\title{
Prevalencia de anticuerpos contra Bartonella henselae en niños, en adolescentes y en una población de riesgo ocupacional en Chile
}

\author{
Marcela Ferrés G ${ }^{1}$, Katia Abarca V 1 , Priscilla Prado $D^{2}$, \\ Luisa Montecinos Pla, Maritza Navarrete $C^{3}$, Pablo A Vial $C^{4}$. \\ Prevalence of Bartonella henselae \\ antibodies in Chilean children, \\ adolescents and veterinary workers
}

Background: Bartonella henselae is the causative agent of catscratch disease. Aim: To study the seroepidemiology of Bartonella henselae in healthy Chilean children and in a population with occupational risk. Material and methods: Serum IgG antibodies were determined by indirect fluorescence technique in 181 children and adolescents and in 107 technical and professional workers involved in the care of cats. Samples with titers equal to or greater than 64 were considered positive. Results: Twenty four (13.3\%) children and $11(10.3 \%)$ occupational risk subjects were seropositive. No significant differences by age and gender were observed. Conclusions: Assuming that seroprevalence indicates level of exposure to Bartonella henselae, these results suggest that this infection is endemic in Chile and, for this reason, the best antibody titer to diagnose acute cat-scratch disease should be higher than the figure recommended by the Centers for Disease Control in the in United States (Rev Méd Chile 2006; 134: 863-7).

(Key w ords: Antibodies, bacterial; Bartonella henselae; Cat-scratch disease)

Recibido el 8 de junio, 2005. Aceptado el 26 de diciembre, 2005.

Proyecto financiado por Fondecyt №1990124.

${ }^{1}$ Laboratorio de Infectología y Virología Molecular, Pontificia Universidad Católica de Chile, Santiago de Chile. ${ }^{2}$ Servicio de Pediatría, Hospital Padre Hurtado, ${ }^{3}$ Instituto de Microbiología Clínica, Universidad Austral de Chile, Valdivia, Chile. ${ }^{4}$ Facultad de Medicina Clínica Alemana y Universidad del Desarrollo de Chile.

aBioquímico

$\mathrm{B}$ artonella henselae es reconocida como el agente etiológico de la enfermedad por arañazo de gato (EAG) desde 1994. Desde entonces, este agente se ha asociado a un creciente espectro

Correspondencia a: Dra. Marcela Ferrés G. Laboratorio de Infectología y Virología Molecular, Pontificia Universidad Católica de Chile. Marcoleta 391, 4⿳0 piso, Santiago, Chile. Fono: 56-2-354 6823. Fax: 56-2-638 7457. E mail: mferres@med.puc.cl de manifestaciones clínicas. La infección se ha descrito a nivel mundial y existen algunas encuestas serólogicas que informan su prevalencia en diversas poblaciones. Estudios realizados en niños, han encontrado prevalencias de $15 \%$ en Grecia y 18,5\% en Canadá1,2; en adultos, las cifras varían entre 3 y $6 \%$ en Estados Unidos de Norteamérica (EE.UU) continental, Hawai, España, Tailandia y Japón ${ }^{3-7}$ hasta $37 \%$ en Canadá ${ }^{8}$. 
Estudios en poblaciones de riesgo ocupacional en EE.UU y Japón informan cifras entre 7 y $15 \% 9,10$. En dueños de gatos, se han encontrado prevalencias de $29 \%$ en España ${ }^{5}$ y de $18 \%$ en un pequeño grupo en Chile ${ }^{11}$.

Estudios realizados por investigadores del Centro de Control de Enfermedades de Atlanta (CDC), EE.UU, han estimado que títulos de IgG específica anti-Bartonella henselae iguales o mayores a 64 (los títulos se reportan como el inverso de la dilución del suero), se correlacionan bien con enfermedad aguda, ya que se encuentran en más de $85 \%$ de los casos clínicos sintomáticos de EAG y sólo en 3\% de los controles sanos ${ }^{3}$. Por ello, en ese país, se considera que la presencia de IgG anti- Bartonella henselae en título de 64 en un sujeto sin manifestaciones clínicas de EAG es indicativo de exposición previa a la bacteria y es por tanto, el nivel de corte usado para estudios de seroprevalencia.

En Chile, la EAG no es una enfermedad de notificación obligatoria, por lo que su relevancia a nivel de salud pública nacional no ha sido determinada. Hasta la fecha, sólo se han publicado en nuestro medio pequeñas series clínicas de infecciones por Bartonella hensela $e^{12-14}$. En consideración a que el diagnóstico de EAG, tanto en sus manifestaciones clásicas como atípicas, ha ido en aumento en nuestro medio, es necesario mejorar el conocimiento epidemiológico de esta infección en Chile.

El objetivo primario de este estudio fue determinar la prevalencia de anticuerpos IgG antiBartonella henselae en dos poblaciones chilenas: niños y adolescentes sanos y sujetos con exposición ocupacional a gatos. Los objetivos secundarios fueron identificar posibles diferencias por edad y género; comparar las prevalencias obtenidas en ambas poblaciones; e investigar posibles factores asociados a la infección en los sujetos con riesgo laboral.

\section{PoBLACIÓN Y MÉTODO}

Niños y adolescentes. Se analizaron sueros de 181 niños sanos residentes en Santiago. Los sueros pertenecían a una colección tomada durante 19881990 para un estudio seroepidemiológico de hepatitis $\mathrm{A}^{15}$. Se obtuvo autorización de la Unidad de Bioética del Ministerio de Salud para utilizar estos sueros. Las muestras fueron seleccionadas al azar, de un total de 681 sueros de sujetos entre $1 \mathrm{a}$ 19 años y procesadas en forma ciega, utilizando un código que informaba edad y género de cada caso estudiado. No se contó con información epidemiológica relevante para EAG como el antecedente de contacto con gatos.

Población de riesgo ocupacional. Se invitó a participar a médicos veterinarios y técnicos veterinarios que trabajan en tres bioterios universitarios y diez clínicas veterinarias que atienden animales pequeños, de las ciudades de Santiago y Valdivia. Ciento siete sujetos aceptaron participar (95\% de los contactados). Se les entrevistó mediante un cuestionario y se les extrajo $5 \mathrm{~mL}$ de sangre venosa para la detección de anticuerpos.

Métodos diagnósticos. Serología: Se determinó la presencia de anticuerpos séricos IgG específicos anti-Bartonella henselae por técnica de inmunofluorescencia indirecta (IFI), utilizando un kit comercial (Focus ${ }^{\mathrm{TM}}$ ). Esta prueba utiliza células Vero E6 infectadas con Bartonella henselae, las que fijadas sobre un portaobjeto son incubadas con el suero a analizar. La unión del anticuerpo del paciente con el antígeno bacteriano se visualiza bajo microscopio de fluorescencia, mediante observación de fluorescencia verde manzana brillante sobre los bacilos fijados en el portaobjeto. La sensibilidad esperada del ensayo es de 88$95 \% 16$. Las muestras fueron procesadas en el Laboratorio de Infectología y Virología Molecular de la Pontificia Universidad Católica de Chile, siguiendo las instrucciones del fabricante. Los sueros se procesaron en dilución basal de 1:64 y aquellos que resultaron positivos se diluyeron hasta determinar sus títulos. En cada procedimiento se usó un control negativo y uno positivo ${ }^{17}$.

Entrevista ocupacional. Se aplicó un cuestionario a la población de riesgo laboral solicitando información demográfica, actividad ocupacional y antecedentes epidemiológicos de exposición a gatos. Se incluyeron las siguientes variables como posibles factores asociados a infección: tipo de trabajo (veterinarios $\mathrm{v} / \mathrm{s}$ auxiliares técnicos) y antecedente de accidentes con gatos (arañazo, mordedura, herida infectada, linfadenopatía regional). 
Estadística. Se utilizó programa Epi 6 y prueba estadística Fisher de 2 colas para comparar las variables en estudio para cada uno de los subgrupos (positivos y negativos para anticuerpos antiBartonella henselae).

\section{RESULTADOS}

Niños y adolescentes. Se analizaron un total de 181 muestras de individuos, cuya edad fluctuó entre 2 y 17 años (promedio 6,5), 49,7\% sexo masculino. Se encontró anticuerpos IgG anti-Bartonella henselae en 24 sujetos (13,3\%). En 21 de ellos (11,6\% del total), el título de anticuerpos fue de 64 y en 3 $(1,7 \%)$ fue de 256 . No hubo diferencias por género: $14 / 90(15,5 \%)$ de los hombres y 10/91 (11\%) de las mujeres resultaron seropositivos ( $p$ $>0,1)$. La prevalencia por grupo etario se presenta en la Figura 1. Se observa tendencia al aumento de la seroprevalencia con la edad, aunque esta variación no fue significativa al comparar cada uno de los grupos de edad analizados $(p>0,1)$.
Población de riesgo ocupacional. Se analizaron un total de 107 muestras, 58,8\% hombres, edad promedio 29 años (rango 18 a 63). Se encontró anticuerpos IgG anti-Bartonella henselae en 11 (10,3\%), todos con títulos de 64 . La prevalencia por género mostró tendencia a mayor positividad en mujeres, sin alcanzar valor estadístico: 4/63 (6,3\%) de los hombres y $7 / 44(15,9 \%)$ de las mujeres resultaron positivos $(p=0,1)$. La Tabla 1 resume la relación entre prevalencia y las variables estudiadas. Los datos sugieren una mayor prevalencia en auxiliares técnicos que en veterinarios y en individuos que habían sufrido arañazo y mordedura por gatos. Sin embargo, las diferencias no resultaron estadísticamente significativas. Finalmente, la presencia de anticuerpos anti-Bartonella henselae fue significativamente mayor en individuos con historia de herida (arañazo o mordedura) con signos de infección. Las otras variables estudiadas no fueron estadísticamente significativas.

Comparación entre ambas poblaciones. La prevalencia de anticuerpos anti-Bartonella henselae no

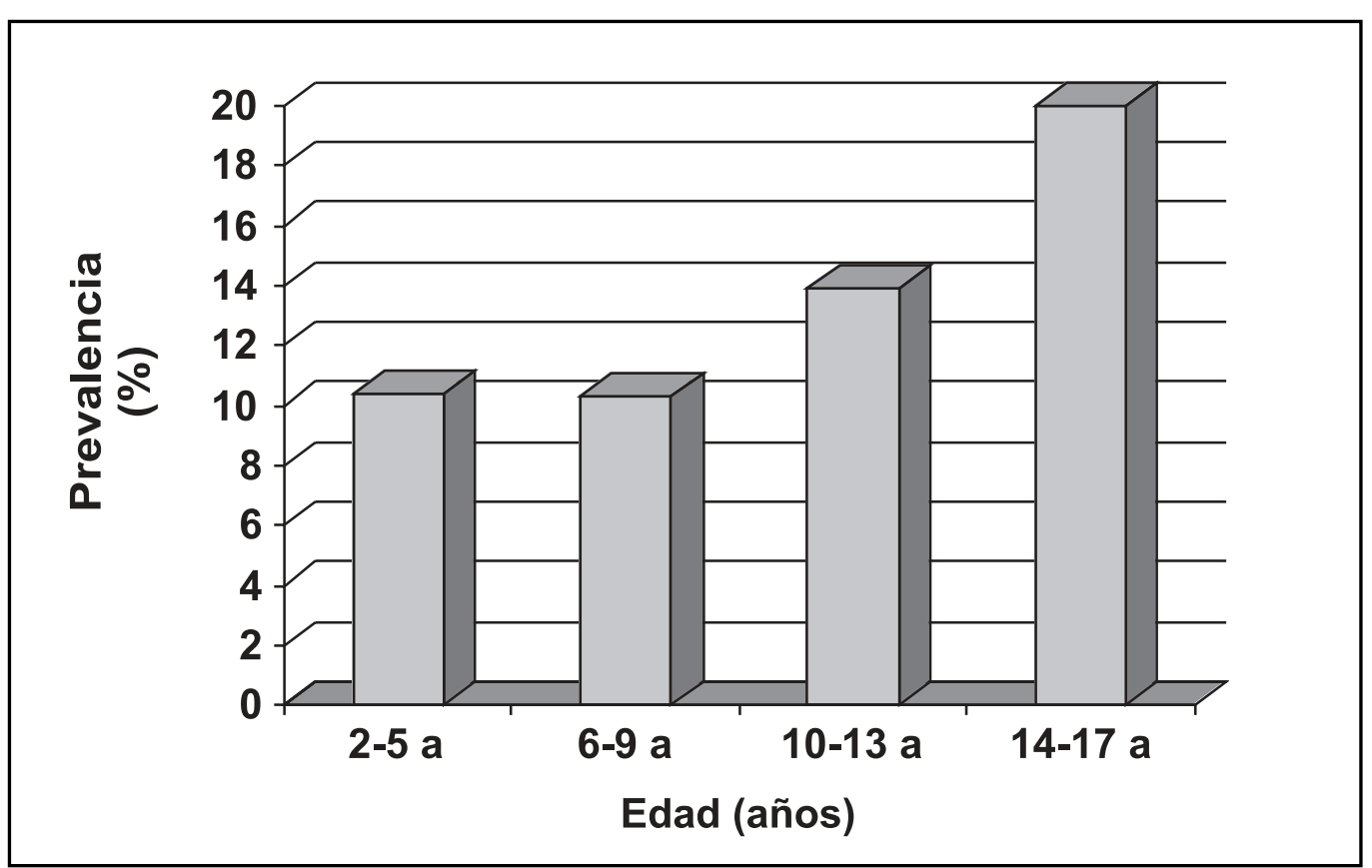

Figura 1. Distribución por edad de la seroprevalencia de Bartonella henselae en población pediátrica, Santiago, Chile $(\mathrm{n}=181)$. 
Table 1. Seroprevalencia de Bartonella henselae según factores de riesgo, en 107 sujetos con riesgo ocupacional

\begin{tabular}{|lccc|}
\hline Características de los sujetos & Positivos/total & $\%$ & $\mathrm{p}^{* *}$ \\
\hline Veterinarios & $5 / 76$ & 6,6 & 0,056 \\
Auxiliares técnicos & $6 / 31$ & 19,4 & $>0,1$ \\
Antecedente de accidente con gatos* & $11 / 98$ & 11,2 & $>0,1$ \\
Antecedente de arañazo de gato & $2 / 39$ & 5,1 & 0,060 \\
Antecedente de arañazo y mordedura de gato & $9 / 58$ & 15,5 & 0,038 \\
Antecedente de herida infectada & $5 / 21$ & 23,8 & $>0,1$ \\
Antecedente de linfadenopatía sugerente de EAG & $2 / 7$ & 28,6 & \\
\hline
\end{tabular}

*Rasguño o mordedura. **Test de Fisher 2 colas.

difiere en forma estadísticamente significativa entre la población pediátrica estudiada y los sujetos con riesgo ocupacional $(p=0,45)$.

\section{DisCUSIÓN}

Bartonella henselae ha sido reconocida como el agente causal de la EAG, angiomatosis bacilar y peliosis hepática. En este estudio se investigó la prevalencia de exposición a Bartonella henselae, a través de la detección de anticuerpos IgG específicos contra este agente. Los resultados obtenidos en población pediátrica muestran una prevalencia de 13,3\%. Esta cifra es similar a las encontradas en otros estudios realizados en niños en Grecia y Canadá1,2. En forma similar, la prevalencia obtenida en los sujetos con riesgo ocupacional $(10,3 \%)$, se asemeja a otros informes que señalan cifras que oscilan entre 7 y $15 \% 9,10$.

Estos resultados sugieren que la infección por Bartonella henselae es endémica en Chile. Esta observación es concordante con la alta prevalencia de anticuerpos encontrados en gatos (75-95\%) de tres regiones de Chile ${ }^{18}$ y a la apreciación de los pediatras chilenos de un elevado número de casos de EAG en nuestro medio.

La EAG es considerada la primera causa de adenopatía crónica regional en niños en EE.UU, con una incidencia estimada en 22.000 casos anuales ${ }^{19}$, lo que la sitúa como una de las enfermedades zoonóticas más comunes en ese país. Si bien en Chile no se dispone de datos de incidencia de esta infección, la prevalencia encontrada en este estudio sugiere que, muy probablemente, en nuestro país también es una zoonosis de gran importancia.

La mayoría de los estudios realizados en adultos sanos han encontrado anticuerpos anti-Bartonella henselae en alrededor de $5 \%{ }^{3-7}$, en Chile no existen estudios de este tipo. La mayor prevalencia encontrada en las dos poblaciones estudiadas en relación a lo informado en adultos en otros países, sugiere que los niños y el personal de salud veterinaria podrían constituir grupos de riesgo para adquirir esta infección por tener mayor exposición a gatos y mayor niesgo de sufrir accidentes (arañazos o mordeduras) que faciliten la transmisión. Son necesarios nuevos estudios de prevalencia que incluyan adultos chilenos sin contacto ocupacional con gatos para confirmar esta hipótesis.

En los sujetos con riesgo ocupacional, se pudo identificar una asociación estadística entre el antecedente de herida infectada e infección por Bartonella henselae. Otros dos factores, la condición de auxiliares de veterinaria y el antecedente de haber sufrido arañazo y mordedura por gatos, mostraron una tendencia sin alcanzar significancia estadística, probablemente por el pequeño tamaño muestral. Una identificación más completa de los factores de riesgo asociados a infección requerirá de la realización de nuevos estudios con mayor tamaño muestral e idealmente con diseño de cohorte o caso control.

En EE.UU, el diagnóstico de EAG se sustenta en la presencia de anticuerpos IgG anti-Bartonella henselae en títulos de 64 o mayores en una persona con síntomas compatibles; títulos de 64 en población sana son considerados indicativos de infección pasada. El hallazgo de un elevado 
porcentaje $(13,3 \%)$ de niños chilenos asintomáticos con títulos de IgG anti-Bartonella henselae de 64 , sugiere que en nuestro medio, este título no

\section{REFERENCIAS}

1. Antoniou M, Economou I, Wang X, Psaroulaki I, Papadopoulos B, Christidou A et al. Fourteen-year seroepidemiological study of zoonoses in a Greek village. Am J Trop Med Hyg 2002; 66: 80-5.

2. Cimolai N, Benoit L, HiLl A, Lyons C. Bartonella henselae infection in British Columbia: evidence for an endemic disease among humans. Can J Microbiol 2000; 46: 908-12.

3. Regnery R, Olson J, Perkins B, Bibb W. Serological response to Rochalimaea henselae antigen in suspected cat-scratch disease. Lancet 1992; 339: 1443-5.

4. Demers DM, Bass JW, Vincent JM, Person DA, Noyes DK, Staege CM et al. Cat scratch disease in Hawaii: etiology and seroepidemiology. J Pediatric 1995; 127: 23-6.

5. Blanco JR, Oteo Ja, Martínez V, Ramale E, García A, IBARRA V. Seroepidemiology of Bartonella henselae infection in a risk group. Rev Clin Esp 1998; 198: 805-9.

6. Maruyama S, Boonmar S, Morita Y, Sakai T, Tanaka S, YAMAGUCHI F ET AL Seroprevalence of Bartonella henselae and Toxoplasma gondii among healthy individuals in Thailand. J Vet Med Sci 2000; 62: 635-7.

7. Kikuchi E, Maruyama S, Sakai T, Tanaka S, Yamaguchi F, Hagiwara T ET al Serological investigation of Bartonella henselae infections in clinically cat-scratch disease-suspected patients, patients with cardiovascular diseases, and healthy veterinary students in Japan. Microbiol Immunol 2002; 46: 313-6.

8. Sander A, Posselt M, Oberie K, Bredt W. Seroprevalence of antibodies to Bartonella henselae in patients with cat scratch disease and in healthy controls: evaluation and comparison of two commercial serological tests. Clin Diag Lab Immunol 1998; 5: 486-90.

9. Noah DL, Kramer CM, Verbsky MP, Rooney JA, Smith KA, CHILDS JE. Survey of veterinary professionals and other veterinary conference attendees for antibodies to Bartonella henselae and Bartonella quintana. J Am Vet Med Assoc 1997; 210: 342-4.

10. Kumasaka K, ARashima Y, Yanai M, Hosokawa N, Kawano K. Survey of veterinary professionals for antibodies to Bartonella henselae in Japan. Rinsho Byori 2001; 49: 906-10. sería adecuado para diagnosticar infección aguda y que para estos fines debiera usarse un punto de corte mayor.

11. Zaror L, Ernst S, Navarrete M, Baliesteros A, BoroscheCK D, FerRés M ET AL. Detección serológica de Bartonella henselae en gatos en la ciudad de Valdivia, Chile. Arch Med Vet 2002; 34: 103-9.

12. Abarca K, Vial P, Rivera M, García C, Oddó D, Prado P ET AL. Infección por Bartonella henselae en pacientes inmunocompetentes: Enfermedad por arañazo de gato. Rev Méd Chile 1996; 124: 1341-9.

13. Wolff E, Muñoz MP, Zapata C, Ledermann W. Enfermedad por arañazo de gato complicada con compromiso sistémico, osteomielitis vertebral y absceso paravertebral. Rev Chil Infect 2000; 17: 332-9.

14. TÄger M, Zamorano J. Osteomielitis, una manifestación inusual de la enfermedad por arañazo de gato. Rev Chil Infect 2000; 17: 326-31.

15. Fix A, San Martín O, Gauichio L, Vial P, Lagos R. Age-specific prevalence of antibodies to hepatitis A in Santiago, Chile: Risk factors and shift in age of infection among children and young adults. Am J Trop Med Hyg 2002; 66: 628-32.

16. Peter JB, Boyle M, Patnaik M, Hadfield TL, Barka NE, Schwartzman WA et al. Persistent generalized lymphadenopathy and non-Hodgkin's lymphoma in AIDS: association with Rochalimaea henselae infection. Clin Diagn Lab Immunol 1994; 1: 115-6.

17. Viral and Rickettsial Zoonoses Branch, Centers for Disease Control and Prevention. Serodiagnosis of Emerging Infectious Diseases: Bartonella and Ehrlichia Infections. Workshop, February 1-4, 1999.

18. Ferrés M, Abarca K, Godoy P, García P, Palavecino E, MÉndez G et al. Presencia de Bartonella henselae en gatos: cuantificación del reservorio natural y riesgo de exposición humana de esta zoonosis en Chile. Rev Méd Chile 2005; 133: 1465-71.

19. Bass J, Vincent J, Person D. Expanding spectrum of Bartonella infections: Cat Scratch Disease. Pediatric Infect Dis J 1997; 16: 163-9.

\section{Agradecimientos}

Agradecemos a la Dra. Rosanna Lagos, del Centro de Vacunas para el Desarrollo de Santiago, quien nos proporcionó los sueros de la población pediátrica. 